\title{
I005 MODELING VANADIUM BROMOPEROXIDASE: SYNTHESIS, STRUCTURE AND REACTIVITY OF VANADIUM-IMIDAZOLE COMPLEXES.
}

Charles R. Cornman, Jeff Kampf, Myoung Soo Lah and Vincent L. Pecoraro," Department of Chemistry, University of Michigan, Ann Arbor, Michigan, 48109-1055.

Studies of vanadium coordination compounds containing imidazole ligands have been undertaken to understand the relationship between the structure and spectroscopy of vanadium coordination compounds as related to vanadium dependent enzymes [1]. The ligand $\mathrm{H}_{2}$ SALIM [4-(2. salicylideneiminoethyl)imidazole], prepared by the condensation of histamine and salicylaldehyde, reacts with various vanadium starting materials to form several new complexes. These have been structurally characterized and contain imidazole in the vanadium coordination sphere as shown in Figure 1 for VIVO(HSALIM) 2 . Two other complexes, VIVO(HSALIM)SAL and VIVO(HSALIM)Acac, have been crystallographically characterized and contain salicylaldehyde (SAL) and acetylacetonate (Acac) replacing the bidentate HSALIM ligand of $\mathrm{VIV}_{\mathrm{O}}(\mathrm{HSALIM})_{2}$. An acetato complex has been synthesized and is formulated as a dimeric species.

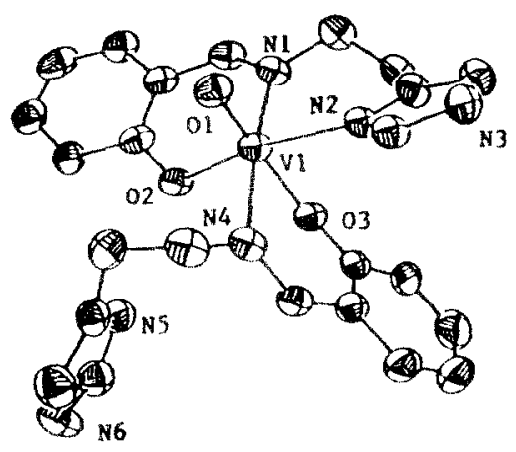

Figure 1. ORTEP diagram of VIVO(HSALIM) 2 .

All four complexes are characterized by axial electron paramagnetic resonance (EPR) spectra. The $g$ and A parameters of the EPR spectra are dependent on proton concentration as shown in Figure 2 for the low field lines of $\mathrm{VIV}^{\mathrm{IV}} \mathrm{O}$ (HSALIM)Acac. Traces $\mathrm{a}, \mathrm{b}$ and $\mathrm{c}$ represent successive additions of acid to form a new protonated species while trace $d$ shows the result of subsequent addition of an equal amount of base indicating the reversibility of the reaction. The trend toward larger hyperfine coupling constant, $\mathrm{A}$, with increased acidity is strikingly similar

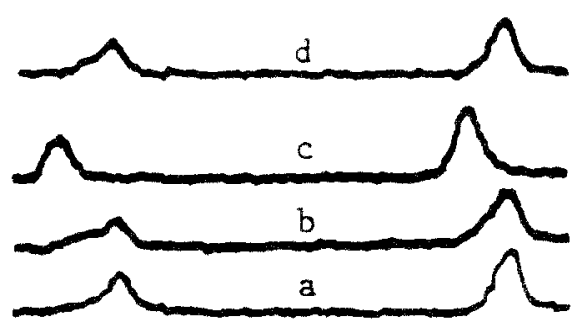

Figure 2. EPR spectra following the acid titration of $V^{I V}$ O(HSALIM)Acac to the observed $\mathrm{pH}$ dependence of the EPR spectra of reduced vanadium bromoperoxidase [2]. These results support the proposal that vanadium bromoperoxidase contains a mixed nitrogen/oxygen coordination sphere.

1. D. Rehder, Angew. Chem. Int. Ed., 30, 148 (1991).

2. E. de Boer, K. Boon and R. Wever, Biochemistry, 27, 1629 (1988). 